\title{
Voltage-Dependent Inactivation of MscS Occurs Independently of the Positively Charged Residues in the Transmembrane Domain
}

\author{
Takeshi Nomura, $^{1,2}$ Masahiro Sokabe, ${ }^{1,3}$ and Kenjiro Yoshimura ${ }^{1,4}$ \\ ${ }^{1}$ International Cooperative Research Project (ICORP)/Solution Oriented Research for Science and Technology (SORST), \\ Cell-Mechanosensing Project, Japan Science and Technology Agency, Nagoya 466-8550, Japan \\ ${ }^{2}$ Department of Rehabilitation, Kyushu Nutrition Welfare University, Kitakyushu 800-029, Japan \\ ${ }^{3}$ Mechanobiology Laboratory, Nagoya University Graduate School of Medicine, Nagoya 466-8550, Japan \\ ${ }^{4}$ Department of Machinery and Control Systems, Shibaura Institute of Technology, Saitama 337-8570, Japan
}

Correspondence should be addressed to Takeshi Nomura; tnomura@knwu.ac.jp

Received 5 September 2016; Accepted 23 November 2016

Academic Editor: Daisuke Miyoshi

Copyright (C) 2016 Takeshi Nomura et al. This is an open access article distributed under the Creative Commons Attribution License, which permits unrestricted use, distribution, and reproduction in any medium, provided the original work is properly cited.

MscS (mechanosensitive channel of small conductance) is ubiquitously found among bacteria and plays a major role in avoiding cell lysis upon rapid osmotic downshock. The gating of MscS is modulated by voltage, but little is known about how MscS senses membrane potential. Three arginine residues (Arg-46, Arg-54, and Arg-74) in the transmembrane (TM) domain are possible to respond to voltage judging from the MscS structure. To examine whether these residues are involved in the voltage dependence of MscS, we neutralized the charge of each residue by substituting with asparagine (R46N, R54N, and R74N). Mechanical threshold for the opening of the expressed wild-type MscS and asparagine mutants did not change with voltage in the range from -40 to $+100 \mathrm{mV}$. By contrast, inactivation process of wild-type MscS was strongly affected by voltage. The wild-type MscS inactivated at +60 to $+80 \mathrm{mV}$ but not at -60 to $+40 \mathrm{mV}$. The voltage dependence of the inactivation rate of all mutants tested, that is, R46N, R54N, R74N, and R46N/R74N MscS, was almost indistinguishable from that of the wild-type MscS. These findings indicate that the voltage dependence of the inactivation occurs independently of the positive charges of R46, R54, and R74.

\section{Introduction}

Various types of mechanosensitive (MS) channels are present in virtually all living organisms and detect forces due to mechanical stimulus such as touch, hearing, turgor, and osmotic change [1-6]. The bacterial MS channels of small $(\mathrm{MscS})$ and large $(\mathrm{MscL})$ conductance are considered to act as a "safety valve" to protect cells from lysis upon osmotic downshock by releasing osmolytes [7-9].

MscS is directly activated by membrane stretch [10] and the gating is modulated by membrane voltage [11-13]. The MscS crystal structure resolved at $3.9 \AA$ shows that MscS is a homoheptamer of a subunit with three TM helices (TM1, TM2, and TM3). A large cytoplasmic vestibule with seven side portals and a distal entrance possibly acts as a molecular prefilter for ion permeation [14-18]. MscS has a conductance of $\sim 1 \mathrm{nS}$ and has a slight preference to anions as the permeable ions $[10,11]$.

MscS shows marked voltage-dependent inactivation under depolarizing conditions [13, 19-22]. Inactivation is facilitated when an electrostatic interaction between TM and cytoplasmic domains is disrupted [21]. The arginine residues at positions 46 and 74 in TM1 and TM2, respectively, of $\mathrm{MscS}$ have been predicted as the candidates for voltage sensor taking into account that the voltage-gated $\mathrm{Na}^{+}, \mathrm{K}^{+}$, and $\mathrm{Ca}^{2+}$ channels have an array of arginine residues especially in the fourth TM segment (S4) that carries most of the gating charge as the voltage sensor [23-27] (Figure 1). Arg-54 in TM1 may also be susceptible to membrane voltage since it is embedded in lipid bilayer in the modeled structure [28]. However, these 


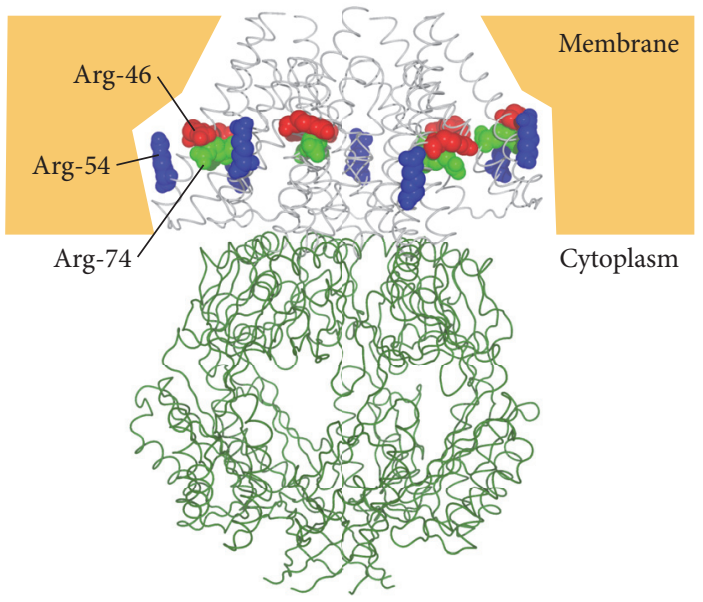

Figure 1: Homoheptameric structure of MscS (2OAU, [30]). The residues 46 (red), 54 (Blue), and 74 (green) are shown in space fill representation. The transmembrane (white) and cytoplasmic (green) domains are shown in ribbon representation.

arginine residues have not been examined in the context of voltage-dependent inactivation [13, 19-21, 29].

In the present study, we investigated whether or not the three charged residues (R46, R54, and R74) in the TM1 and TM2 domains of MscS are involved in the voltagedependent inactivation by using patch-clamp technique and hypoosmotic shock experiment. Our results suggest that voltage-dependent inactivation occurs independently of the positively charged residues in the TM domains of MscS.

\section{Materials and Methods}

The Escherichia coli strain PB111 ( $\Delta m s c S)$ and MJF455 ( $\Delta m s c L \Delta m s c S$ ) were used to host MscS expression in patchclamp and hypoosmotic shock experiments [21, 31]. Sitedirected mutagenesis was performed on the $m s c S$ gene in a pB10b vector by mega-primer PCR method [32]. Successful mutagenesis was verified by DNA sequencing. Mutants were expressed in PB111 or MJF455.

Giant spheroplasts were prepared as described [33]. Briefly, PB111 cells were grown in a modified LB (Luria Bertani) medium containing $0.5 \% \mathrm{NaCl}$ instead of $1 \% \mathrm{NaCl}$ [11] in the presence of cephalexin (final concentration: $0.06 \mathrm{mg} / \mathrm{mL}$ ). After incubation for $1.5 \mathrm{~h}$, IPTG (isopropyl- $\beta$ D-thiogalactoside) was added (final concentration: $1 \mathrm{mM}$ ) to induce MscS expression. The induction time was $10 \mathrm{~min}$. The cells were harvested, digested by lysozyme $(0.2 \mathrm{mg} / \mathrm{mL})$, and collected by centrifugation.

The channel activities of MscS were recorded in the inside-out membrane patch mode of the patch-clamp technique as described previously [32]. The pipette solution contained $200 \mathrm{mM} \mathrm{KCl}, 90 \mathrm{mM} \mathrm{MgCl}_{2}, 10 \mathrm{mM} \mathrm{CaCl}_{2}$, and $5 \mathrm{mM}$ HEPES ( $\mathrm{pH}$ 6.0), whereas the bath solution additionally contained $300 \mathrm{mM}$ sucrose. Negative pressure was applied by using a syringe at various voltages (intracellular potential against extracellular one) ranging from -100 to $+40 \mathrm{mV}$ to obtain the gating threshold of MscS and MscL. For the evaluation of the inactivation process, pressure was controlled using High-Speed Pressure-Clamp apparatus (HSPC1; ALA Scientific Inst. Inc., Westbury, NY) [34], following the protocol by Akitake et al. [13]. Currents were amplified with an AXOPATCH 200B amplifier (Axon Instruments, Foster City, CA), and data were acquired at a sampling rate of $5 \mathrm{kHz}$ with $2 \mathrm{kHz}$ low-pass filtration. The pCLAMP 9 software (Axon Instruments, Foster City, CA) was used for data acquisition and analysis. The MscS/MscL gating threshold ratios were determined by the pressure (proportional to membrane tension) applied through patch pipettes required for the first channel opening of MscS and MscL [32].

Cell viability after hypoosmotic shock was determined by the method described previously $[9,31,35]$. When cell density reached $\mathrm{OD}_{600}=\sim 0.15$ in the minimal medium, IPTG $(1 \mathrm{mM})$ was added to induce expression. After $1 \mathrm{~h}$ of growth, the cells were diluted 1:20 in the prewarmed minimal medium with or without $0.5 \mathrm{M} \mathrm{NaCl}$. A downshock into $0 \mathrm{M} \mathrm{NaCl}$ medium was applied to the cells for $5 \mathrm{~min}$. After the downshock, each sample was spread on LB agar plates containing $1 \mathrm{mM}$ IPTG and incubated overnight at $37^{\circ} \mathrm{C}$ before counting the ratio of colony forming units. The downshock experiment was performed three times for each MscS mutant.

\section{Results}

Candidate arginine residues (R46, R54, and R74) that are possibly involved in voltage sensing were substituted with asparagine to examine the effects of charge neutralization. Thus, we generated three single mutants (R46N, R54N, and $\mathrm{R} 74 \mathrm{~N})$ and one double mutant (R46N/R74N). To examine the effect of these mutations on the gating threshold of MscS, the channel activities were measured while applying negative pressure through a patch pipette to the inside-out membrane patch of the giant spheroplasts expressing the wild-type or mutant MscS. Figure 2(a) shows typical channel activities of the wild-type $\mathrm{MscS}$ at membrane voltage of $+20 \mathrm{mV}$. 


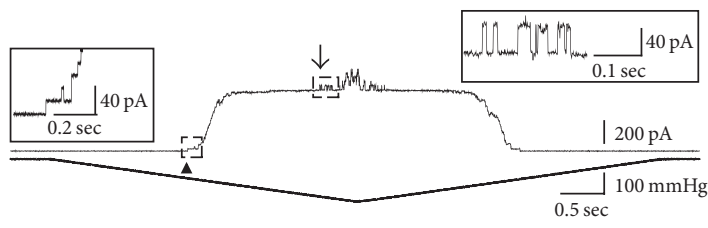

(a)

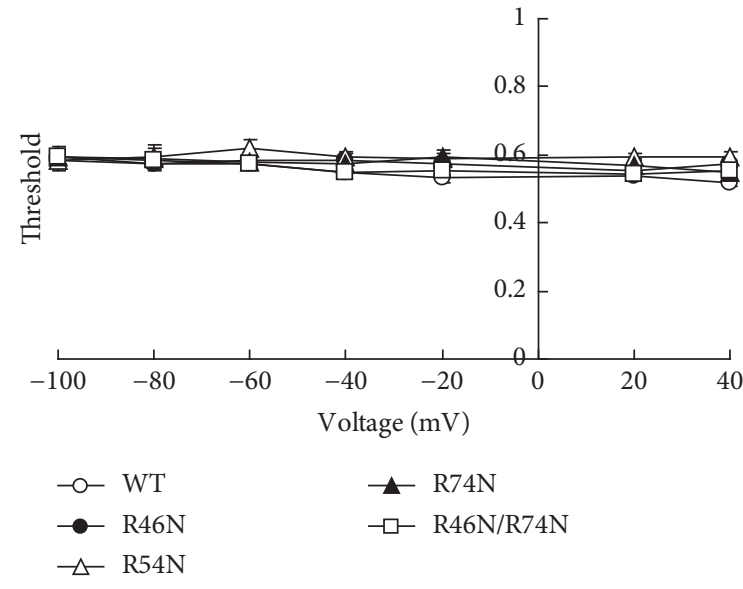

(b)

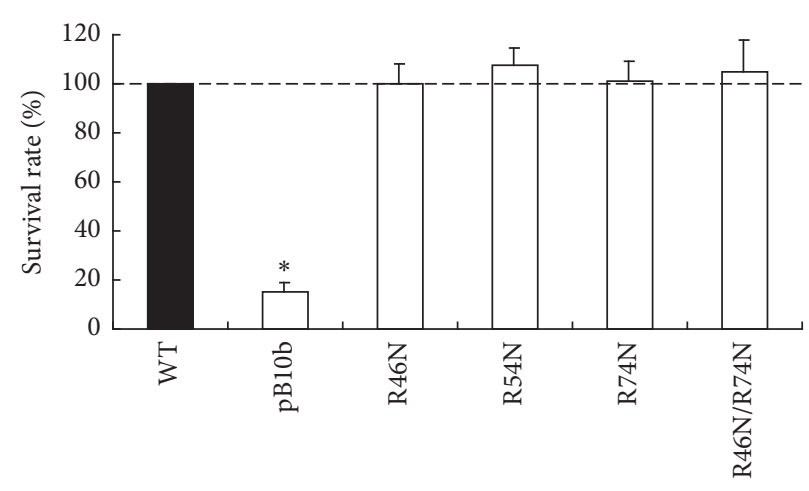

(c)

Figure 2: Characteristics of the wild-type and mutant MscS expressed in PB111 ( $\Delta m s c S$ ) cells. (a) PB11l cells expressing the wild-type MscS. Channel current (upper) and pressure applied through a pipette (bottom) are shown. The insets show the magnification of the MscS and MscL traces indicated by arrowhead and arrow, respectively. Membrane voltage: $+20 \mathrm{mV}$. (b) The gating threshold ratio (MscS/MscL) of the wild-type and mutant MscS at various voltages $(-100$ to $+40 \mathrm{mV})$. Values are expressed as mean \pm SEM (standard error of the mean) $(n=5-$ 7). (c) Effects of hypoosmotic shock on MJF455 ( $\Delta m s c S \Delta m s c L$ ) cells expressing MscS or harboring an empty vector (pB10b) (mean \pm SEM, $n=9)$. No significant difference was observed between the wild-type and mutant MscS. The asterisks indicate significant difference from the wild-type $(p<0.05$ by $t$-test).

Upon increasing the negative pressure, MscS appeared first (arrowhead). The channel conductance was $\sim 1$ nS. Further increase in the negative pressure opened MscL, which has a conductance of $\sim 2.5 \mathrm{nS}$ (arrow). MscS and MscL were assigned based on the conductance and threshold. Using MscL as an internal standard, the threshold of MscS was expressed as the ratio to that of MscL (MscS/MscL). The threshold of the wild-type MscS was constantly $\sim 0.6$ when the membrane (cytoplasmic) potential was scanned between -100 and $+40 \mathrm{mV}$ (Figure 2(b)). The gating threshold of each MscS mutant (R46N, R54N, R74N, and R46N/R74N) was also constant at $\sim 0.6$ in this voltage range.

When $E$. coli cells are exposed to hypoosmotic condition, they avoid cell lysis by opening MscS and MscL. Consistently, most of MJF455 double-knockout ( $\Delta m s c L \Delta m s c S$ ) cells harboring an empty vector (pB10b) did not survive upon hypoosmotic shock from $0.5 \mathrm{M}$ to $0 \mathrm{M} \mathrm{NaCl}$ (Figure 2(c)). Hypotonic shock experiments are advantageous to patch-clamp experiments in that the MscS activity can be assessed under native conditions. When the cells expressing MscS mutants were challenged with hypoosmotic shock, the threshold did not differ statistically from that of wild-type MscS (R46N; $100 \pm 8 \%, \mathrm{R} 54 \mathrm{~N} ; 108 \pm 7 \%, \mathrm{R} 74 \mathrm{~N} ; 101 \pm 8 \%$ and R46N/R74N; $105 \pm 13 \%$; Figure 2(c)), suggesting that the charge neutralization does not alter the MscS activity to a degree at which cell survival is affected.
MscS exhibits prominent voltage-dependent inactivation under depolarizing conditions [13, 19-21]. To test whether the neutralization of the positively charged residues in TM segments modifies the inactivation, we recorded the inactivation at cytoplasmic potentials ranging from -60 to $+80 \mathrm{mV}$. Figure 3(a) illustrates the current traces of channel activities of the wild-type MscS (upper trace). A rapid decrease in channel current was observed at +60 and $+80 \mathrm{mV}$, whereas the decay in current was significantly slow at negative potentials. R46N MscS also inactivated at +60 and $+80 \mathrm{mV}$ but not at negative potentials (Figure 3(a), lower trace). Furthermore, R54N MscS, R74N MscS, and R46N/R74N MscS showed rapid inactivation at positive potentials of +60 and $+80 \mathrm{mV}$ (Figure S1) (see Supplementary Material available online at http://dx.doi.org/10.1155/2016/2401657). Figure 3(b) depicts the relationship between voltage and time constant of inactivation when an exponential function is fitted. All MscS mutants showed rapid current decrease at +60 and $+80 \mathrm{mV}$ at rates similar to the wild-type MscS.

In the above experiment, the initial phase of inactivation was not resolved because it overlapped with the activation phase. To separate the inactivation process from the activation process, we activated $\mathrm{MscS}$ at $-20 \mathrm{mV}$ by applying negative pressure and then switched the membrane potential to $+60 \mathrm{mV}$ (Figure $4(\mathrm{a})$ ). The transition from the full open to closed state fitted best to two exponential functions at $+60 \mathrm{mV}$ 

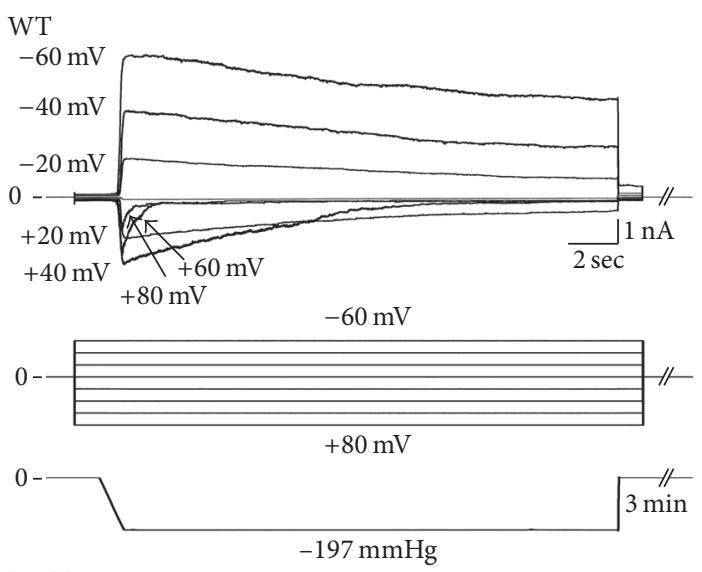

$\mathrm{R} 46 \mathrm{~N}$

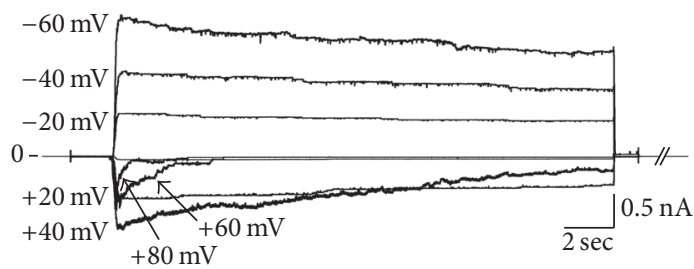

(a)

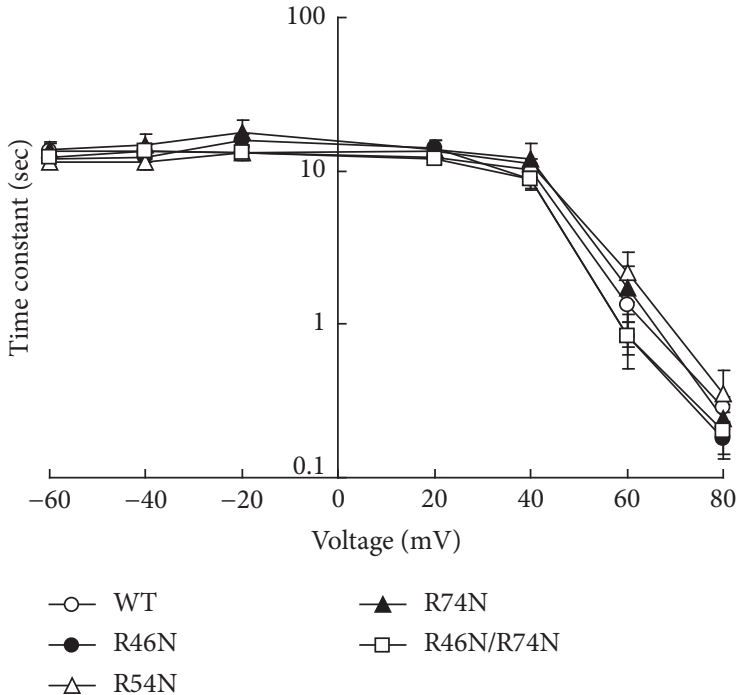

(b)

FIGURE 3: Inactivation of the wild-type and mutant MscS expressed in MJF455 ( $\Delta m s c S \Delta m s c L$ ) cells. (a) Channel currents (upper) of the wildtype and $\mathrm{R} 46 \mathrm{~N} \mathrm{MscS}$ at different voltages ranging from -60 to $+80 \mathrm{mV}$ (middle). Subsaturating negative pressure (bottom) was applied after the change in voltage. (b) The time constant of inactivation at each voltage. Time constant was faster at depolarizing voltages of +60 and $+80 \mathrm{mV}$ (mean \pm SEM, $n=5-9$ ).

in the wild-type MscS: the fast and slow time constants were $\tau=0.28 \pm 0.03 \mathrm{sec}$ and $\tau=0.88 \pm 0.27 \mathrm{sec}$, respectively. This observation indicates that voltage-dependent inactivation process has multiple processes. When the same protocol was applied to the R46N MscS (Figure 4(a) lower trace), R54N MscS, R74N MscS, and R46N/R74N MscS (Fig. S2), the current decayed at rates roughly similar to the wild-type MscS.

Figure 4(b) summarizes the fast and slow time constants of the wild-type and mutant MscS. Either component did not differ significantly between the wild-type and mutant MscS. The above observations indicate that charged residues at TM domains are not involved in the voltage-dependent inactivation process.

\section{Discussion}

An array of positively charged residues in the S4 segment of voltage-gated ion channels is responsible for voltagedependent channel activities [23,24]. Voltage dependence of MscS may also be attributed to the positively charged residues in TM1 and TM2 [27]. In the present study, we investigated the role of arginine residues in TM1 and TM2 in the voltagedependent inactivation of MscS. The channel properties of $\mathrm{MscS}$ mutants were examined using patch-clamp technique and cell viability test. Against our prediction, we found that the neutralization of positive charges in TM1 and TM2 has little impact on the voltage dependence of MscS inactivation.

The voltage sensor in voltage-gated sodium, potassium, and calcium channels contains a conserved pattern of 6-7 positively charged residues (Arg or Lys) in their S4 segment [36]. These charges move on a change in the electric potential gradient across the TM domain, resulting in the channel gating. The gating charge of $\mathrm{Kv}$ channels has been estimated to be about 12-13 per channel [37]. On the other hand, the opening of MscS is only weakly voltage-dependent and thus the gating charge of MscS is estimated to be as small as about 0.8 per channel [13]. By contrast, the inactivation of MscS is more dependent on voltage and two positive charges are proposed to be transferred [13].

The movement of gating charges is of crucial importance for understanding the mechanism of voltage-dependent inactivation of MscS. The positively charged residues R46, R54, and R74 are predicted to be close to the cytoplasmic surface of the lipid bilayer in the closed resting state. Generally, when a membrane potential shifts from hyperpolarizing to depolarizing condition, TM helices with positive charges move toward the extracellular side [36]. On the other hand, TM1 and TM2 of MscS interact with membrane lipid and the hydrophobic residues at both ends of TM1 and TM2 provide lipid-protein interaction important for mechanosensitivity [31]. Therefore, even if the electrical field is changed, TM1 and TM2 may move little in the direction perpendicular to membrane because of the tight lipid-protein interactions at both ends of the TM helices. This speculation argues against the idea that TM1 and TM2 move on depolarization but is consistent with the present result.

In a molecular dynamics simulation (MD) study, neutralization of R46 and R74 reduces the hydration of the pore and 


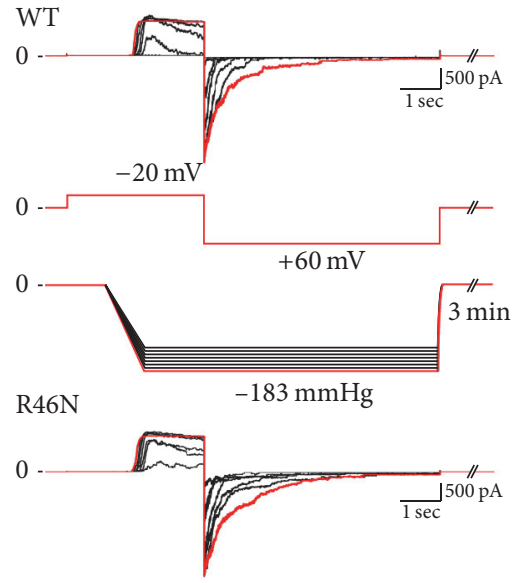

(a)

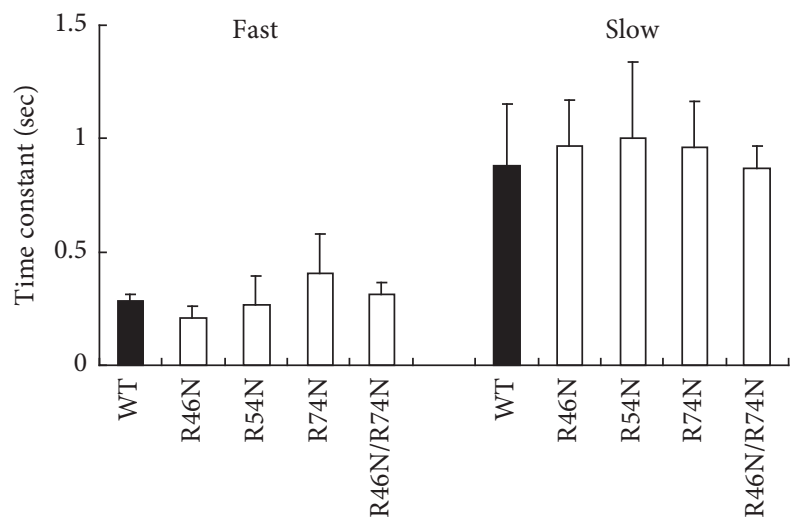

(b)

FIGURE 4: Rate of inactivation in the wild-type and mutant MscS expressed in MJF455 ( $\Delta m s c S \Delta m s c L$ ) cells. (a) Macroscopic currents of wildtype (upper) and R46N MscS (bottom). Voltage was changed from -20 to $+60 \mathrm{mV}$ (upper middle) in the presence of pressure (lower middle). Red traces show the traces at the pressure where MscS was activated fully ( $-183 \mathrm{mmHg}$ in wild-type and $-203 \mathrm{mmHg}$ in R46N MscS). (b) Time constant of fast and slow components of the wild-type and mutant MscS (mean \pm SE, $n=5-7$ ) as determined by fitting current traces to two-exponential functions.

results in loss of conductivity, although they are distant from the pore [38]. However, our results show that neutralizing these positively charged residues does not influence the conductance of MscS. The MD study detected movement of TM1 and TM2 at $\pm 100 \mathrm{mV}$ but not at smaller voltage; the movement was reduced greatly in R46 mutants. This finding also does not fit well our data that inactivation occurs at +60 and $+80 \mathrm{mV}$ and that $\mathrm{R} 46 \mathrm{~N}$ MscS inactivates like the wildtype MscS. These discrepancies may be because the crystal structure used in the MD study is different from the structure in the lipid bilayer or under membrane stretch.

A variety of models of MscS inactivation process have been proposed: (1) shrinking of the cytoplasmic vestibule [39], (2) kink formation between the pore-lining helix TM3a and TM3b, which connects the gate and the cytoplasmic vestibule [29], and (3) electrostatic interaction between the upper surface of the cytoplasmic vestibule and the loop that connects the TM1-TM2 helices [21]. However, none of these models incorporate the charged residues in TM1 and TM2. Thus, we speculate that potential gradient is present not only in TM domain but also in the cytoplasmic vestibule and that the cytoplasmic vestibule is responsible for the voltage dependence of the inactivation process of MscS.

In conclusion, we have shown that the neutralization of the arginine residues in TM1 and TM2 does not influence the stretch activation and voltage-dependent inactivation of MscS. We also found that voltage-dependent inactivation process has two (fast and slow) components. The structural bases of these components are yet to be determined.

\section{Competing Interests}

The authors declare no conflict of interests.

\section{Acknowledgments}

This work was supported by a Grant-in-Aid from the Ministry of Education, Culture, Sports, Science, and Technology, Japan (26460300 to Takeshi Nomura).

\section{References}

[1] A. Anishkin and C. Kung, "Microbial mechanosensation," Current Opinion in Neurobiology, vol. 15, no. 4, pp. 397-405, 2005.

[2] J. H. Naismith and I. R. Booth, "Bacterial mechanosensitive channels-MscS: evolution's solution to creating sensitivity in function," Annual Review of Biophysics, vol. 41, no. 1, pp. 157177,2012

[3] C. Kung, "A possible unifying principle for mechanosensation," Nature, vol. 436, no. 7051, pp. 647-654, 2005.

[4] C. D. Cox, Y. Nakayama, T. Nomura, and B. Martinac, "The evolutionary 'tinkering' of MscS-like channels: generation of structural and functional diversity," Pflugers Archiv European Journal of Physiology, vol. 467, no. 1, pp. 3-13, 2014.

[5] O. P. Hamill and B. Martinac, "Molecular basis of mechanotransduction in living cells," Physiological Reviews, vol. 81, no. 2, pp. 685-740, 2001.

[6] E. Perozo, "Gating prokaryotic mechanosensitive channels," Nature Reviews Molecular Cell Biology, vol. 7, no. 2, pp. 109-119, 2006.

[7] P. Blount, M. J. Schroeder, and C. Kung, "Mutations in a bacterial mechanosensitive channel change the cellular response to osmotic stress," The Journal of Biological Chemistry, vol. 272, no. 51, pp. 32150-32157, 1997.

[8] B. Ajouz, C. Berrier, A. Garrigues, M. Besnard, and A. Ghazi, "Release of thioredoxin via the mechanosensitive channel MscL during osmotic downshock of Escherichia coli cells," The Journal of Biological Chemistry, vol. 273, no. 41, pp. 26670-26674, 1998. 
[9] N. Levina, S. Tötemeyer, N. R. Stokes, P. Louis, M. A. Jones, and I. R. Booth, "Protection of Escherichia coli cells against extreme turgor by activation of MscS and MscL mechanosensitive channels: identification of genes required for MscS activity," The EMBO Journal, vol. 18, no. 7, pp. 1730-1737, 1999.

[10] S. Sukharev, "Purification of the small mechanosensitive channel of Escherichia coli (MscS): the subunit structure, conduction, and gating characteristics in liposomes," Biophysical Journal, vol. 83, no. 1, pp. 290-298, 2002.

[11] B. Martinac, M. Buechner, A. H. Delcour, J. Adler, and C. Kung, "Pressure-sensitive ion channel in Escherichia coli," Proceedings of the National Academy of Sciences of the United States of America, vol. 84, no. 8, pp. 2297-2301, 1987.

[12] P. Koprowski and A. Kubalski, "Voltage-independent adaptation of mechanosensitive channels in Escherichia coli protoplasts," Journal of Membrane Biology, vol. 164, no. 3, pp. 253-262, 1998.

[13] B. Akitake, A. Anishkin, and S. Sukharev, "The 'dashpot' mechanism of stretch-dependent gating in MscS," Journal of General Physiology, vol. 125, no. 2, pp. 143-154, 2005.

[14] R. B. Bass, P. Strop, M. Barclay, and D. C. Rees, "Crystal structure of Escherichia coli MscS, a voltage-modulated and mechanosensitive channel," Science, vol. 298, no. 5598, pp. 1582-1587, 2002.

[15] P. Koprowski and A. Kubalski, "C termini of the Escherichia coli mechanosensitive ion channel (MscS) move apart upon the channel opening," Journal of Biological Chemistry, vol. 278, no. 13, pp. 11237-11245, 2003.

[16] S. Miller, M. D. Edwards, C. Ozdemir, and I. R. Booth, "The closed structure of the MscS mechanosensitive channel. Crosslinking of single cysteine mutants," Journal of Biological Chemistry, vol. 278, no. 34, pp. 32246-32250, 2003.

[17] S. Miller, W. Bartlett, S. Chandrasekaran, S. Simpson, M. Edwards, and I. R. Booth, "Domain organization of the MscS mechanosensitive channel of Escherichia coli," The EMBO Journal, vol. 22, no. 1, pp. 36-46, 2003.

[18] C. D. Cox, T. Nomura, C. S. Ziegler, A. K. Campbell, K. T. Wann, and B. Martinac, "Selectivity mechanism of the mechanosensitive channel MscS revealed by probing channel subconducting states," Nature Communications, vol. 4, article no. 2137, 2013.

[19] V. Vásquez and E. Perozo, "Voltage dependent gating in MscS," Biophysical Journal, vol. 86, p. 545A, 2004.

[20] M. Sotomayor, V. Vásquez, E. Perozo, and K. Schulten, "Ion conduction through MscS as determined by electrophysiology and simulation," Biophysical Journal, vol. 92, no. 3, pp. 886-902, 2007.

[21] T. Nomura, M. Sokabe, and K. Yoshimura, "Interaction between the cytoplasmic and transmembrane domains of the mechanosensitive channel MscS," Biophysical Journal, vol. 94, no. 5, pp. 1638-1645, 2008.

[22] I. Rowe, A. Anishkin, K. Kamaraju, K. Yoshimura, and S. Sukharev, "The cytoplasmic cage domain of the mechanosensitive channel MscS is a sensor of macromolecular crowding," The Journal of General Physiology, vol. 143, no. 5, pp. 543-557, 2014.

[23] L. M. Mannuzzu, M. M. Moronne, and E. Y. Isacoff, "Direct physical measure of conformational rearrangement underlying potassium channel gating," Science, vol. 271, no. 5246, pp. 213216, 1996.
[24] A. Cha and F. Bezanilla, "Characterizing voltage-dependent conformational changes in the Shaker $\mathrm{K}^{+}$channel with fluorescence," Neuron, vol. 19, no. 5, pp. 1127-1140, 1997.

[25] S. K. Aggarwal and R. MacKinnon, "Contribution of the S4 segment to gating charge in the Shaker $\mathrm{K}^{+}$channel," Neuron, vol. 16, no. 6, pp. 1169-1177, 1996.

[26] S.-A. Seoh, D. Sigg, D. M. Papazian, and F. Bezanilla, "Voltagesensing residues in the S2 and S4 segments of the Shaker $\mathrm{K}^{+}$ channel," Neuron, vol. 16, no. 6, pp. 1159-1167, 1996.

[27] F. Bezanilla and E. Perozo, "Structural biology: force and voltage sensors in one structure," Science, vol. 298, no. 5598, pp. $1562-$ $1563,2002$.

[28] A. Anishkin, B. Akitake, and S. Sukharev, "Characterization of the resting MscS: modeling and analysis of the closed bacterial mechanosensitive channel of small conductance," Biophysical Journal, vol. 94, no. 4, pp. 1252-1266, 2008.

[29] B. Akitake, A. Anishkin, N. Liu, and S. Sukharev, "Straightening and sequential buckling of the pore-lining helices define the gating cycle of MscS," Nature Structural and Molecular Biology, vol. 14, no. 12, pp. 1141-1149, 2007.

[30] S. Steinbacher, R. Bass, P. Strop, and D. C. Rees, "Structures of the prokaryotic mechanosensitive channels MscL and MscS," Current Topics in Membranes, vol. 58, pp. 1-24, 2007.

[31] T. Nomura, M. Sokabe, and K. Yoshimura, "Lipid-protein interaction of the MscS mechanosensitive channel examined by scanning mutagenesis," Biophysical Journal, vol. 91, no. 8, pp. 2874-2881, 2006.

[32] K. Yoshimura, A. Batiza, M. Schroeder, P. Blount, and C. Kung, "Hydrophilicity of a single residue within MscL correlates with increased channel mechanosensitivity," Biophysical Journal, vol. 77, no. 4, pp. 1960-1972, 1999.

[33] P. Blount, S. I. Sukharev, P. C. Moe, B. Martinac, and C. Kung, Mechanosensitive Channels of Bacteria, vol. 294 of Mehods in Enzymology, 1999.

[34] S. R. Besch, T. Suchyna, and F. Sachs, "High-speed pressure clamp," Pflugers Archiv European Journal of Physiology, vol. 445, no. 1, pp. 161-166, 2002.

[35] K. Yoshimura, T. Nomura, and M. Sokabe, "Loss-of-function mutations at the rim of the funnel of mechanosensitive channel MscL," Biophysical Journal, vol. 86, no. 4, pp. 2113-2120, 2004.

[36] F. Bezanilla, "The voltage sensor in voltage-dependent ion channels," Physiological Reviews, vol. 80, no. 2, pp. 555-592, 2000.

[37] N. E. Schoppa, K. McCormack, M. A. Tanouye, and F. J. Sigworth, "The size of gating charge in wild-type and mutant Shaker potassium channels," Science, vol. 255, no. 5052, pp. 1712$1715,1992$.

[38] S. A. Spronk, D. E. Elmore, and D. A. Dougherty, "Voltagedependent hydration and conduction properties of the hydrophobic pore of the mechanosensitive channel of small conductance," Biophysical Journal, vol. 90, no. 10, pp. 3555-3569, 2006.

[39] W. Grajkowski, A. Kubalski, and P. Koprowski, "Surface changes of the mechanosensitive channel MscS upon its activation, inactivation, and closing," Biophysical Journal, vol. 88, no. 4, pp. 3050-3059, 2005. 

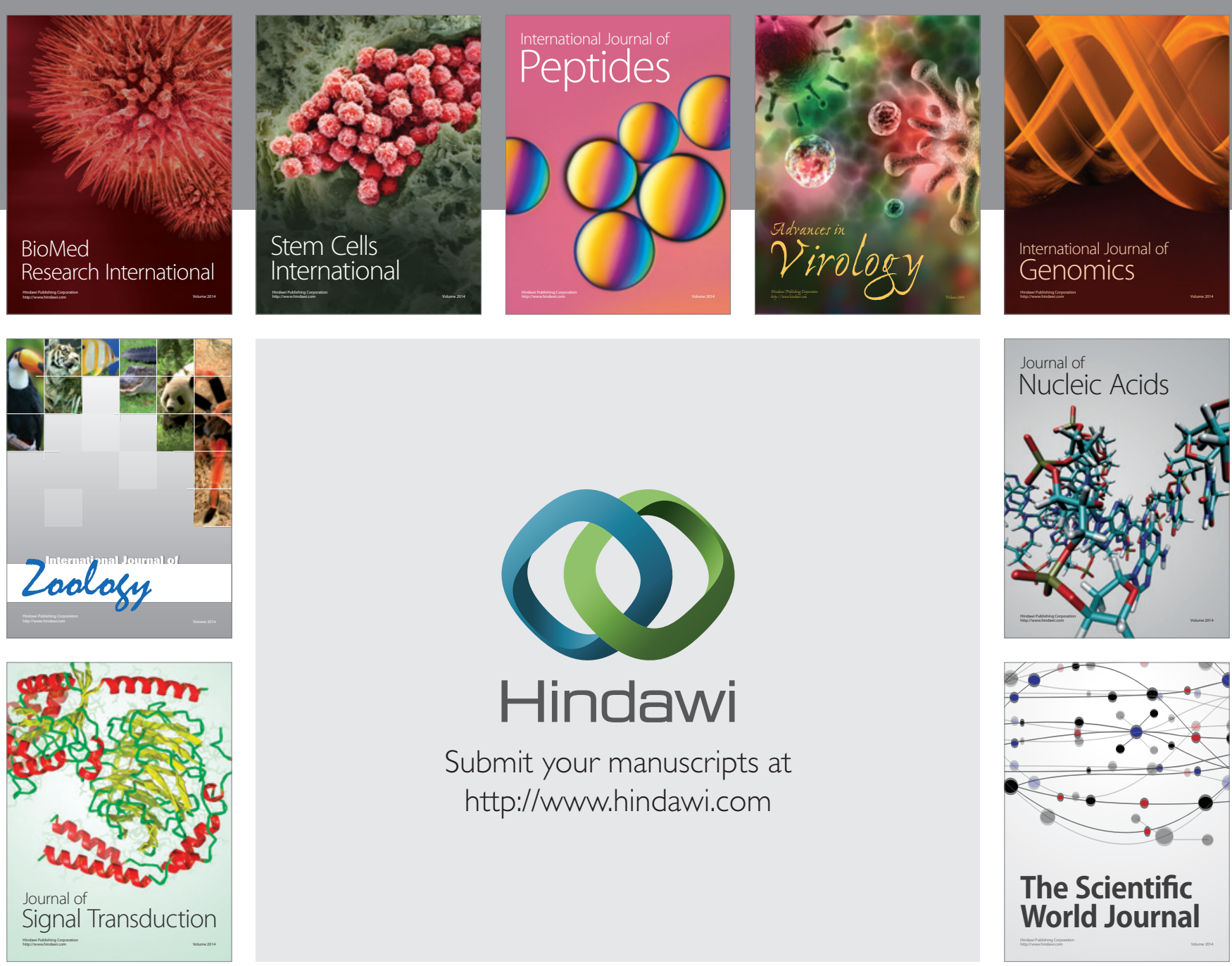

Submit your manuscripts at

http://www.hindawi.com
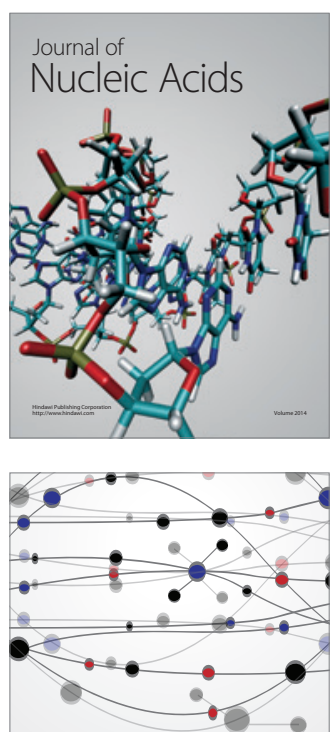

The Scientific World Journal
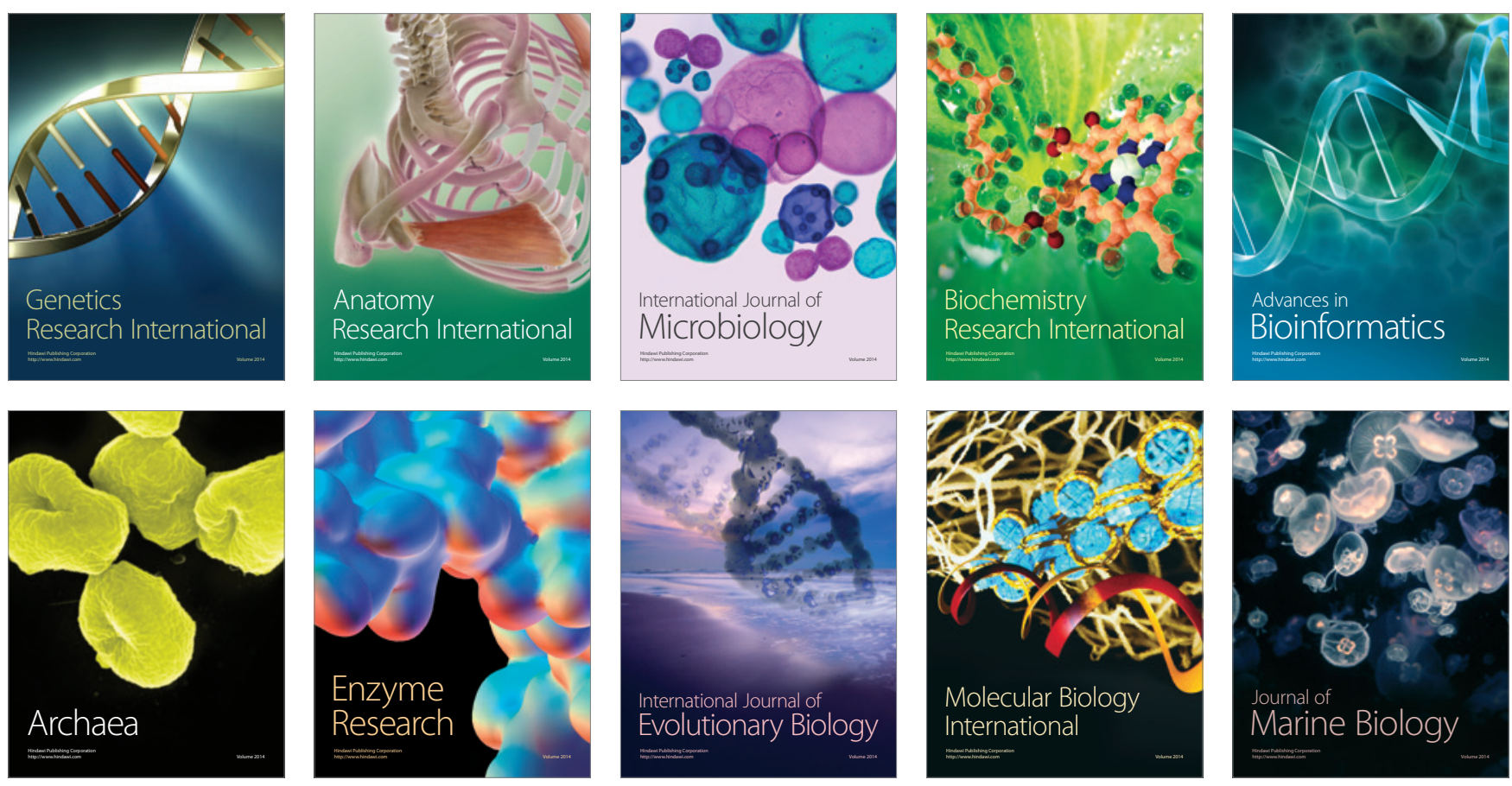